\title{
The Effects of Propellant Slosh Dynamics on the Solar Dynamics Observatory
}

\author{
Paul Mason, Scott R. Starin ${ }^{1}$ \\ Goddard Space Flight Center, Code 595, Greenbelt, MD, 20771
}

\begin{abstract}
The Solar Dynamics Observatory (SDO) mission, which is part of the Living With a Star program, was successfully launched and deployed from its Atlas V launch vehicle on February 11, 2010. SDO is an Explorer-class mission now operating in a geosynchronous orbit (GEO). The basic mission is to observe the Sun for a very high percentage of the 5-year mission (10-year goal) with long stretches of uninterrupted observations and with constant, high-data-rate transmission to a dedicated ground station located in White Sands, New Mexico. A significant portion of SDO's launch mass was propellant, contained in two large tanks. To ensure performance with this level of propellant, a slosh analysis was performed. This paper provides an overview of the SDO slosh analysis, the on-orbit experience, and the lessons learned.
\end{abstract}

SDO is a three-axis controlled, single fault tolerant spacecraft. The attitude sensor complement includes sixteen coarse Sun sensors, a digital Sun sensor, three two-axis inertial reference units, two star trackers, and four guide telescopes. Attitude actuation is performed either using four reaction wheels or eight thrusters, depending on the control mode, along with single main engine which nominally provides velocity-change thrust. The attitude control software has five nominal control modes: three wheel-based modes and two thruster-based modes. A wheel-based Safehold running in the Attitude Control Electronics (ACE) box improves the robustness of the system as a whole. All six modes are designed on the same basic proportional-integral-derivative attitude error structure, with more robust modes setting their integral gains to zero.

To achieve and maintain a geosynchronous orbit for a $2974 \mathrm{~kg}$ spacecraft in a cost effective manner, the SDO team designed a high-efficiency propulsive system. This bi-propellant design includes a $100 \mathrm{lbf}$ main engine and eight $5 \mathrm{lbf}$ attitude control thrusters. The main engine provides high specific impulse for the maneuvers to attain GEO, while the smaller Attitude Control System (ACS) thrusters manage the disturbance torques of the larger main engine and provide the capability for much smaller orbit adjustment burns. SDO's large solar profile produces a large solar torque disturbance and momentum buildup. This buildup drives the frequency of momentum unloads via ACS thrusters. SDO requires $1409 \mathrm{~kg}$ (which is approximately half the launch mass) of propellant to achieve and maintain the GEO orbit while performing the momentum unloads for 10 years.

For missions requiring large amounts of propellant for orbit insertion/maintenance or momentum unload, it is imperative that slosh dynamics, the motion of any free liquid propellant surface inside the propellant tanks and its impact on the spacecraft, are understood. This propellant motion can result in periodic disturbance forces and torques on a spacecraft or launch vehicle. These slosh effects must be accounted for in the control mode design. A poor controller design can excite the slosh dynamics, which can adversely impact the performance and stability of the spacecraft. Due to the higher levels of forces and torques, slosh effects are more prevalent during the thruster-based modes (DeltaH and DeltaV). Therefore, a preflight slosh analysis and simulation should be performed with the thruster modes.

The purpose of the preflight slosh analysis, which had two levels of fidelity, was to quantify and understand the slosh dynamics. The initial slosh analysis examined the dynamics of slosh in a bare tank configuration, i.e. no Propellant Management Device (PMD). An important product of

\footnotetext{
${ }^{1}$ Attitude Control Systems Engineering Branch, NASA Goddard Space Flight Center, Greenbelt, Maryland 20771 USA. 
the bare tank analysis was an initial equivalent mechanical slosh model. SDO's equivalent mechanical slosh model, a point-mass pendulum, was used in simulations to determine the impact of slosh on the attitude dynamics during various maneuvers. For conservatism, the worst case errors and uncertainties were used in these simulations. The simulation results were used to verify and/or refine requirements as well as identify problems and investigate possible solutions, which can include modifications to the design. For SDO, the resulting slosh dynamics were the driving factor in the redesign of the propellant tanks to include a PMD. The proposed PMD would greatly increase the damping and reduce the center of mass motion, which should improve the stability and performance.

After the addition of the PMD, a more refined Computational Fluid Dynamics (CFD) slosh analysis was performed by the vendor. This high fidelity analysis modeled the fluid dynamics in the PMD tank. Even though the simulation was very accurate, it required significant computational effort and specialized knowledge, limiting the ability of the SDO project to access fluid dynamics simulations at will. Furthermore, it was very difficult to incorporate most of these models into closed-loop simulations of the overall spacecraft and its environment. In order to observe the effects of the slosh dynamics with a PMD, the equivalent mechanical model was updated and used to determine the impact of slosh on the controller performance and stability. In addition, various limits, thresholds, error bars, and the settling burn time, which is part of the maneuver design, were updated based on the second slosh analysis results.

After a successful launch and Sun Acquisition, SDO performed a system checkout, which included an engineering burn. During the engineering burn, which consisted of a 20 second ACS thruster-only settling burn and a 60 second main engine burn, there were no expected or observed slosh dynamics due a high fill fraction level $(94.65 \%)$. The initial Geosynchronous Transfer Orbit (GTO) maneuver plan consisted of five Apogee Motor Firing (AMF) maneuvers using the main engine followed by three Trim Motor Firing (TMF) maneuvers that only used the ACS thrusters. The first AMF maneuver performed as expected. After the maneuver, both tanks were at an $82 \%$ fill fraction level. Thirty eight seconds into the second AMF maneuver, the momentum levels exceeded $20 \mathrm{Nms}$ and tripped a Fault Detection and Correction (FDC) limit, which aborted the burn and transitioned the spacecraft into Sun Acquisition mode. After the Sun Acquisition mode placed the spacecraft into a Sun safe attitude, a tiger team was assembled to investigate the cause of the aborted burn. While the tiger team was investigating the cause of the abort, the rest of the SDO team revised the GTO maneuver plan to use ACS thrusters only, a planned contingency. After a lot of hard work, the cause of the anomaly was determined to be slosh. The tiger team determined that a longer settling burn of four minutes was needed along with higher momentum limits and an update to the DeltaV structural filter parameters to make the filter a unity passthrough. The DeltaV mode structural filter, used for modal suppression, added a delay into the system and diminished the controller's ability to compensate for the slosh. Once these solutions were validated in simulation, the appropriate changes were made in the flight software and the flight dynamics team designed a new maneuver plan that incorporated the main engine. The resulting four main engine maneuvers all performed as expected.

As a result of this experience, the SDO ACS team has continued to investigate the dynamics of slosh. This continuing investigation and the flight experience have resulting in the following lessons learned: First, the settling burn, which was defined by the geyser slosh mode, needs to be a function of the settling time of several modes. Second, the structural filter, which ensures modal suppression, is not always required during coarse pointing thruster modes. Third, after processing the data from all of the burns, it was determined that slosh was observable at all fill fractions and the equivalent mechanical models may not be sufficient to capture the coupled fluid-structural dynamics. Finally, the tank configuration, in particular the center mass location of the propellant relative to the center of mass of the spacecraft, does play a significant part in the fluid structural interaction associated with slosh. The lessons learned in this work will aid other projects in ensuring that slosh does not have an adverse impact on the mission. 